Article

\title{
Nonlocal initial value problems for Katugampola-Caputo type fractional differential equations on time scales
}

\author{
Alagarsamy Nandhini ${ }^{1}$, Devaraj Vivek ${ }^{1, *}$ and Elsayed M. Elsayed ${ }^{2,3}$ \\ 1 Department of Mathematics with Computer Applications, Sri Ramakrishna College of Arts and Science (Formerly \\ SNR sons College), Coimbatore-641 006, India; chikunandhu17@gmail.com (A.N) \\ 2 Department of Mathematics, Faculty of Science, Mansoura University, Mansoura 35516, Egypt; \\ emmelsayed@yahoo.com) \\ 3 Department of Mathematics, Faculty of Science, King Abdulaziz University,Jeddah 21589, Saudi Arabia \\ * Correspondence: peppyvivek@gmail.com
}

Received: 04 August 2018; Accepted: 10 December 2018; Published: 8 February 2019.

\begin{abstract}
In this paper, we study Katugampola fractional differential equations (FDEs) with nonlocal conditions on time scales. By means of standard fixed point theorems, some new sufficient conditions for the existence of solutions are established.
\end{abstract}

Keywords: Nonlocal initial value problem, existence, fixed point, time scales.

MSC: 26A33, 34A08, 45G05.

\section{Introduction}

$\mathbf{T}$ he theory of FDEs has attracted attention of many researchers because of its wide applications in biology, medicine and in other applied fields, see for example [1-4] and references therein. Throughout this paper $(X,\|\cdot\|)$ will be a Banach space, and $I=[0, T], T>0$, a compact interval in $\mathbb{R}$. Let $C=C([0, T], X)$ be the Banach space of all continuous functions $[0, T] \rightarrow X$ endowed with the topology of uniform convergence (the norm in this space will be denoted by $\|\cdot\|_{c}$ ).

In this work we consider the following Cauchy problem for the FDEs with nonlocal conditions on time scales

$$
\begin{aligned}
{ }^{\rho} \Delta_{t_{0}}^{q} x(t) & =f(t, x(t)), \quad t \in I, \\
x(0)+g(x) & =x_{0},
\end{aligned}
$$

where $0<\mathrm{q}<1$.

The system (1),(2) is equivalent to

$$
x(t)=x_{0}-g(x)+\frac{\rho^{1-q}}{\Gamma(q)} \int_{0}^{t}\left(t^{\rho}-s^{\rho}\right)^{q-1} s^{\rho-1} f(s, x(s)) \Delta s
$$

Recent studies of FDEs on time scales are done by Ahmadkhanlu in his papers [5]. The reader may also consult [6-8].

As indicated in Bashir's pioneering paper [2], the nonlocal condition $x(0)+g(0)=x_{0}$ can be applied in physics with better effect than the classical Cauchy problem with initial condition $x(0)=x_{0}$. For instance the author used

$$
g(x)=\sum_{i=1}^{p} c_{i} x\left(t_{i}\right)
$$

where $c_{i}=1,2, \ldots, p$ are given constants and $0<t_{1}<t_{2}<\ldots t_{p} \leq T$. To describe the diffusion phenomenon of a small amount in a transparent tube, the Cauchy problem allows the additional measurement at $t_{i}, i=1,2, \ldots . p$. We adopt some ideas from [9].

We investigate in our paper the Cauchy problem (1),(2) with the following assumptions: 
(H1) $f: \mathbb{R} \times X \rightarrow X$ is jointly continuous.

(H2) $\|f(t, x)-f(t, y)\| \leq L\|x-y\|, \forall t \in \mathbb{R}, x, y \in X$.

(H3) $g: C \rightarrow X$ is continuous and $\|g(x)-g(y)\| \leq b\|x-y\|, \forall x, y \in C$.

\section{Existence results}

We are now ready to present our results.

Theorem 1. Under assumptions (H1) and (H2), if $b<\frac{1}{2}$ and $L \leq \frac{\Gamma(q+1)}{2 T^{q}}$, then the equations (1) and (2) has a unique solution .

Proof. Define $F: C \rightarrow C$ by

$$
(F x)(t)=x_{0}-g(x)+\frac{\rho^{(1-q)}}{\Gamma(q)} \int_{0}^{t}\left(t^{\rho}-s^{\rho}\right)^{(q-1)} f(s, x(s)) \Delta s
$$

choose $r \geq 2\left(\left\|x_{0}\right\|+G+\frac{M T^{q}}{\Gamma(q+1)}\right)$, and let $\sup _{t \in I}\|f(t, 0)\|=M$. Then it is easy to see that $F B_{r} \subset B_{r}$ where $B_{r}=$ $x \in C:\|x\| \leq r$.

So let $x \in B_{r}$ and set $G=\sup _{x \in C}\|g(x)\|$. Then we get

$$
\begin{aligned}
\|F x(t)\| & \leq\left\|x_{0}\right\|+G+\frac{\rho^{(1-q)}}{\Gamma(q)} \int_{0}^{t}\left(t^{\rho}-s^{\rho}\right)^{q-1} s^{\rho-1}\|f(s, x(s))\| \Delta s \\
& \leq\left\|x_{0}\right\|+G+\frac{\rho^{1-q}}{\Gamma(q)} \int_{0}^{t}\left(t^{\rho}-s^{\rho}\right)^{q-1} s^{\rho-1}(\|f(s, x(s))-f(s, o)\|+\|f(s, 0)\|) \Delta s \\
& \leq\left\|x_{0}\right\|+G+(L r+M) \frac{1}{\Gamma(q)} \int_{0}^{t}\left(t^{\rho}-s^{\rho}\right)^{q-1} \Delta s \\
& \leq\left\|x_{0}\right\|+G+(L r+M) \frac{T^{q}}{\Gamma(q+1)} \leq r
\end{aligned}
$$

by the choice of $L$ and $r$. Now take $x, y \in C$, then we get

$$
\begin{aligned}
\|(F x)(t)-(F x)(t)\| & \leq\|g(x)-g(y)\|+\frac{\rho^{1-q}}{\Gamma(q)} \int_{0}^{t}\left(t^{\rho}-s^{\rho}\right)^{q-1} s^{\rho-1}\|f(s, x(s))-f(s, y(s))\| \Delta s \\
& \leq \Omega_{b, L, T, q, \rho}\|x-y\|
\end{aligned}
$$

where $\Omega_{b, L, T, q, \rho}=\left(b+\frac{L T^{\rho q}}{\rho^{q} \Gamma(q+1)}\right)$ depends only on the parameters of the problem and since $\Omega_{b, L, T, q, \rho} \leq 1$, the result follows in view of the contraction mapping principle.

Theorem 2. Let $M$ be a nonempty convex subset of the Banach space X. Let $A, B$ be two operators such that

1. $A x+B y \in M$ whenever $x, y \in M$,

2. A is compact and continuous,

3. $B$ is a contraction mapping,

then there exists $Z \in M$ such that $Z=A x+B z$.

Theorem 3. Assume the conditions (H1)and (H3) holds, $b<1$ and

(H4) $\|f(t, x)\| \leq \mu(t), \quad \forall(t, x) \in I \times X$, where $\mu \in L^{1}\left(I, \mathbb{R}^{+}\right)$,

then the equations (1) and (2) has at least one solution on I.

Proof. Choose $r \geq\left\|x_{0}\right\|+G+\frac{T^{\rho q}\|\mu\|_{L^{1}}}{\rho^{q} \Gamma(q+1)}$ and consider $B_{r}: x \in C:\|x\| \leq r$. Now define on $B_{r}$ the operators $A$ and $B$ as

$$
\begin{aligned}
& (A x)(t)=\frac{\rho^{1-q}}{\Gamma(q)} \int_{0}^{t}\left(t^{\rho}-s^{\rho}\right)^{q-1} s^{\rho-1} f(s, x(s)) \Delta s, \text { and } \\
& (B x)(t)=x_{0}-g(x)
\end{aligned}
$$


If $x, y \in B_{r}$ then $A x+B y \in B_{r}$. Indeed

$$
\|A x+B y\| \leq\left\|x_{0}\right\|+G+\frac{T^{\rho q}\|\mu\|_{L^{1}}}{\rho^{q} \Gamma(q+1)} \leq r .
$$

By $(H 3)$, it is also clear that $B$ is a contraction mapping for $b<1$. Since $x$ is continuous, then $(A x)(t)$ is continuous in view of (H1). Note that $A$ is uniformly bounded on $B_{r}$. Hence we have

$$
\|(A x)(t)\| \leq \frac{T^{\rho q}\|\mu\|_{L^{1}}}{\rho^{q} \Gamma(q+1)} .
$$

Now, we prove that $(A x)(t)$ is equicontinuous. Let $t_{1}, t_{2} \in I$ and $x \in B_{r}$. Using the fact that $f$ is bounded on the compact set $I \times B_{r}$ (thus $\left.\sup _{(t, x) \in I \times B_{r}}\|f(t, x)\|=c_{0}<\infty\right)$, we get

$$
\begin{aligned}
& \left\|A x\left(t_{1}\right)-A x\left(t_{2}\right)\right\| \\
& =\left\|\frac{\rho^{1-q}}{\Gamma(q)} \int_{0}^{t_{1}}\left(t_{1}^{\rho}-s^{\rho}\right)^{q-1} s^{\rho-1} f(s, x(s)) \Delta s-\frac{\rho^{1-q}}{\Gamma(q)} \int_{0}^{t_{2}}\left(t_{2}^{\rho}-s^{\rho}\right)^{q-1} s^{\rho-1} f(s, x(s)) \Delta s\right\| \\
& \leq \frac{\rho^{1-q}}{\Gamma(q)}\left\|\int_{0}^{t_{1}}\left[\left(t_{1}^{\rho}-s^{\rho}\right)^{q-1}-\left(t_{2}^{\rho}-s^{\rho}\right)^{q-1}\right] s^{\rho-1} f(s, x(s)) \Delta s+\int_{t_{1}}^{t_{2}}\left(t_{2}^{\rho}-s^{\rho}\right)^{q-1} s^{\rho-1} f(s, x(s)) \Delta s\right\| \\
& \leq \frac{C_{0} \rho^{1-q}}{\Gamma(q)}\left(\int_{0}^{t_{1}}\left|\left(t_{1}^{\rho}-s^{\rho}\right)^{q-1}-\left(t_{2}^{\rho}-s^{\rho}\right)^{q-1}\right| s^{\rho-1} \Delta s+\int_{t_{1}}^{t_{2}}\left(t_{2}^{\rho}-s^{\rho}\right)^{q-1} s^{\rho-1} \Delta s\right) .
\end{aligned}
$$

For $q<1,\left(t_{1}^{\rho}-s^{\rho}\right)^{q-1} \geq\left(t_{2}^{\rho}-s^{\rho}\right)^{q-1}$, we have

$$
\begin{aligned}
\int_{t_{0}}^{t_{1}}\left|\left(t_{1}^{\rho}-s^{\rho}\right)^{q-1}-\left(t_{2}^{\rho}-s^{\rho}\right)^{q-1}\right| s^{\rho-1} \Delta s & =\int_{t_{0}}^{t_{1}}\left[\left(t_{1}^{\rho}-s^{\rho}\right)^{q-1}-\left(t_{2}^{\rho}-s^{\rho}\right)^{q-1}\right] s^{q-1} \Delta s \\
& =\frac{1}{\rho q}\left(t_{1}^{\rho} q-t_{2}^{\rho} q\right)+\frac{1}{\rho q}\left(t_{2}^{\rho}-t_{1}^{\rho}\right)^{q} \\
& =\frac{1}{\rho q}\left(t_{2}^{\rho}-t_{1}^{\rho}\right)^{q} .
\end{aligned}
$$

If $q>1,\left(t_{1}^{\rho}-s^{\rho}\right)^{q-1} \leq\left(t_{2}^{\rho}-s^{\rho}\right)^{q-1}$, we have

$$
\begin{aligned}
\int_{t_{0}}^{t_{1}}\left|\left(t_{1}^{\rho}-s^{\rho}\right)^{q-1}-\left(t_{2}^{\rho}-s^{\rho}\right)^{q-1}\right| s^{\rho-1} \Delta s & =\int_{t_{0}}^{t_{1}}\left[\left(t_{1}^{\rho}-s^{\rho}\right)^{q-1}-\left(t_{1}^{\rho}-s^{\rho}\right)^{q-1}\right] s^{\rho-1} \Delta s \\
& =\frac{1}{\rho q}\left(t_{2}^{\rho} q-t_{1}^{\rho} q\right)-\frac{1}{\rho q}\left(t_{2}^{\rho}-t_{1}^{\rho}\right)^{q} \\
& =\frac{1}{\rho q}\left(t_{2}^{\rho} q-t_{1}^{\rho} q\right) \\
\left\|(A x)\left(t_{1}\right)-(A x)\left(t_{2}\right)\right\| & \leq \frac{2 C_{0}}{\rho^{q} \Gamma(q+1)}\left(t_{2}^{\rho}-t_{1}^{\rho}\right)^{q}, \quad q \leq 1 \\
\leq & \frac{C_{0}}{\rho^{q} \Gamma(q+1)}\left[\left(t_{2}^{\rho}-t_{1}^{\rho}\right)^{q}+\left(t_{2}^{\rho q}-t_{1}^{\rho q}\right)\right], \quad q>1,
\end{aligned}
$$

which does not depend on $x$, so $A\left(B_{r}\right)$ is relatively compact. By the Arzela-Ascoli Theorem, $A$ is compact. We now conclude the result of the theorem based on the Krasnoselkii's theorem above.

Author Contributions: All authors contributed equally to the writing of this paper. All authors read and approved the final manuscript.

Conflicts of Interest: "The authors declare no conflict of interest."

\section{References}

[1] Balachandran, K., \& Trujillo, J. J. (2010). The nonlocal Cauchy problem for nonlinear fractional integrodifferential equations in Banach spaces. Nonlinear Analysis: Theory, Methods E Applications, 72(12), 4587-4593. 
[2] Bashir, A., \& Sivasundaram, S. (2008). Some existence results for fractional integro-differential equations with nonlocal conditions. Communications in Applied Analysis, 12, 107-112.

[3] NŠGuérékata, G. M. (2009). A Cauchy problem for some fractional abstract differential equation with non local conditions. Nonlinear Analysis: Theory, Methods E Applications, 70(5), 1873-1876.

[4] Richard, H. (2014). Fractional calculus: an introduction for physicists. World Scientific.

[5] Ahmadkhanlu, A., \& Jahanshahi, M. (2012). On the existence and uniqueness of solution of initial value problem for fractional order differential equations on time scales. Bulletin of the Iranian Mathematical Society, 38(1), 241-252.

[6] Katugampola, U. N. (2014). A new approach to generalized fractional derivatives. Bull. Math. Anal. Appl, 6(4), 1-15.

[7] Katugampola, U. N. (2011). New approach to a generalized fractional integral. Applied Mathematics and Computation, 218(3), 860-865.

[8] Katugampola, U. N. (2014). Existence and uniqueness results for a class of generalized fractional differential equations. arXiv preprint arXiv:1411.5229.

[9] Vivek, D., Kanagarajan, K., \& Harikrishnan, S. (2007). Existence and uniqueness results for pantograph equations with generalized fractional derivative. Journal of Nonlinear Analysis and Applications (ISPACS). 